\title{
Probability of Dying in Each of the Competing Risks under Bimorbid Condition
}

\author{
Kalpana Singh ${ }^{1,2}$ and Suddhendu Biswas ${ }^{1}$ \\ ${ }^{1}$ Department of Statistics, Amity Institute of Applied Science (AIAS), Amity University, Noida 201301, India \\ ${ }^{2}$ Public Health Foundation of India, Sector 44, Plot 47, Gurgaon, Haryana 122002, India
}

Correspondence should be addressed to Kalpana Singh; nkalpanasingh@gmail.com

Received 5 December 2016; Revised 2 March 2017; Accepted 2 April 2017; Published 13 April 2017

Academic Editor: Shesh N. Rai

Copyright ( 2017 Kalpana Singh and Suddhendu Biswas. This is an open access article distributed under the Creative Commons Attribution License, which permits unrestricted use, distribution, and reproduction in any medium, provided the original work is properly cited.

\begin{abstract}
The motivation of this study is to explore the respective probability of dying in either of the risks while a person has dual or more competing risks. The problem requires evolution of a model describing the probability of dying in either of risks while a person is exposed to competing risk of two or more diseases. This is obtained by choice of a model which is bivariate negative binomial distribution for obtaining the number of deaths (assuming the deaths occur in the last trial). A Bayesian method has also been used for estimating probability of dying in heart disease and chronic kidney disease in the presence of diabetes, hypertension, and stroke or heart disease with chronic kidney disease. For the analysis of the same we have used CARRS (cardiometabolic risk reduction in south Asia) surveillance data, a multistage cluster random sampling technique.
\end{abstract}

\section{Introduction}

Gumbell (1960) described the earliest and the simplest bivariate exponential distribution. This method has received applications in many areas, including competing risks, extreme values, failure times, regional analyses of precipitation, and reliability.

Downton [1] described the bivariate exponential distribution arising from "shocks" causing various types of failure to components which have geometric distributions. It has received applications in queueing systems and hydrology and has been used as a model for Wold's Markov dependent processes, intensity and duration of rainfall, and height of water waves.

Marshall and Olkin [2] estimated the bivariate exponential distribution in which $X$ and $Y$ are the lifetimes of two components subject to three kinds of shocks; these shocks are assumed to be governed by independent Poisson processes with parameters $\theta 1, \theta 2$, and $\theta 3$, according as whether the shock applies to component 1 only, component 2 only, or both the components. The distribution has received wide applicability in nuclear reactor safety, competing risks, reliability, and quantal response contexts.

Biswas and Noor [3] investigated a method for classifying unknown causes of death due to two competing risks into assignable causes.

The real cause of death for a person, otherwise suffering from two major risks (heart disease with hypertension, heart disease with diabetes, heart disease with chronic kidney disease, diabetes with hypertension, stoke with hypertension, stroke with diabetes, chronic kidney disease with diabetes, and chronic kidney disease with hypertension), is very difficult to estimate and remains unclassified. The purpose of the present paper is to devise a suitable probability model describing the effect of two such competing risks as $R 1$ or $R 2$ and obtain the expected proportion of such unclassified cases suffering from both the conditions $R 1$ and $R 2$ and dying either in $R 1$ or $R 2$. It has been tacitly assumed that while both the risks due to $R 1$ and $R 2$ may be operating at the time of death of a person, really the death in actuality occurs, principally due to either $R 1$ or $R 2$. Further, the development of the model is in the line of a risk model and we assume that 
death is caused ultimately on account of a single risk of either $R 1$ or $R 2$. Biswas and Noor [3] have investigated the same type of problem with hypothetical data with several parameters in the model. However, in this study some parameters were found redundant because of availability of data.

The approach followed by Biswas and Noor [3] was based on number of shocks corresponding to two competing risks ( $R 1$ and $R 2$ ) and the number of shocks causing death by either of the two shocks taken as parameters. The shock due to risk $R 1$ occurs with Poisson intensity and the effect of shock lasts for a fixed period (dead time) during which no further shock due to $R 1$ or $R 2$ may occur. But, in our model, we have not considered the effect of shock, which lasts for a fixed period, during which no further shocks occur due to $R 1$ or $R 2$.

The number of shocks for each of the competing risks that may cause death varies from individual to individual, information of which was readily available from the CARRS Cohort dataset. Therefore, we could avoid redundant parameter estimation. Hence our model may be considered as an extension of earlier Biswas and Noor paper.

Recently McCormick et al. [4] investigated the same type of problem "probabilistic cause-of-death assignment using verbal autopsies." The present application using the Bayesian method is more or less based on the same approach.

\section{Study Design}

As stated earlier, the present study is based on the baseline data from the cross-sectional survey of the three CARRS Study sites conducted between October 2010 and 2011 with follow-up for the investigation of mortality through December 2014. The duration of the follow-up was 3.5 years. The CARRS (cardiometabolic risk reduction in south Asia) Study design was adapted from the WHO STEPS model [5] to assess the prevalence and incidence of cardiometabolic diseases (CMDs) and their risk factors. Details of the CARRS Study design have been published [6]. In brief, participants were selected in each of the three cities (Chennai, New Delhi, and Karachi) using a multistage cluster sampling technique [7]. Municipal subdivisions, wards in Chennai and Delhi and census enumeration blocks in Karachi, were treated as the primary sampling units. Households in selected subdivisions were then manually listed and mapped. The manual household listing was used to randomly select households with equal opportunity for selection. From each randomly selected household, one eligible man and one eligible woman (aged $20+$, nonpregnant, and not bedridden) were selected. For households with more than two eligible adults of the same sex, the "Kish method" [5] (all eligible participants from the household were ranked according to age in decreasing orders (males followed by females); participants were selected using KISH table identifying the last digit of household and number of eligible participants) was applied to randomly select one individual to be included in the study.

\section{Data Collection}

Data were collected from the households through interviews in local languages and standardized clinical examinations as well as blood sample collection either at local camps (Chennai, Delhi) or at home (Karachi). In the three cities, collective baseline response rate was $94.7 \%$ for questionnaire completion and $84.3 \%$ for specimen collection. Data were gathered by trained field teams using standardized techniques [6].

3.1. Definition of Chronic Conditions. In the present study, chronic conditions including hypertension, diabetes, chronic kidney disease, heart disease, and stroke were considered. These medical conditions were defined as follows: hypertension (self-report of prior diagnosis or measured blood pressure of 140/90 mmHg or above); diabetes (self-report of prior diagnosis or fasting plasma glucose of $\geq 126 \mathrm{mg} / \mathrm{dL}$ or HbAlc of $\geq 6.5$ ); heart disease/stroke (self-report of prior diagnosis); CKD (self-report of prior diagnosis). All participant had already had two or more risks at the beginning of the study.

We further classified participants into two-way joint distribution of the specified chronic conditions, that is, heart disease with hypertension, heart disease with diabetes, heart disease with chronic kidney disease, diabetes with hypertension, stroke with hypertension, stroke with diabetes, chronic kidney disease with diabetes, and chronic kidney disease with hypertension.

3.2. Development of the Model. Let $R_{1}$ and $R_{2}$ be two competing risks in order.

(I) $p=$ probability of occurrence of $R_{1}$.

(II) $q=$ probability of occurrence of $R_{2}$, where $p+q=1$.

(III) $M_{1}$ and $M_{2}$ represent the number of trials where the individuals were exposed to the risks $R_{1}$ and $R_{2}$, respectively.

(IV) $\theta=$ probability that a particular risk of first or second type is fatal leading to death.

(V) $1-\theta=$ probability of surviving in $R_{1}$ and $R_{2}$.

(VI) $\chi$ is the probability of dying in either $R_{1}$ or $R_{2}$ without considering that the risks are competing.

(VII) $N_{1}=$ number of death cases exposed to the risk of $R_{1}$ or $R_{2}$ while the death might have occurred either due to $R_{1}$ or $R_{2}$, the real cause being unknown.

(VIII) $N_{2}=$ number of death cases exposed to risk of $R_{1}$ or $R_{2}$ while the real cause is unknown.

$N=N_{1}+N_{2}=$ size of the population.

(IX) $\chi=N_{1} /\left(N_{1}+N_{2}\right)$.

Then we have

$$
N_{1}=N \sum_{x=0}^{M^{\prime}} \sum_{r=0}^{M}\left(\begin{array}{c}
x+r-1 \\
r-1
\end{array}\right)[(1-\theta) p]^{r-1}[(1-\theta) q]^{x}[p(\theta)]
$$




$$
\begin{aligned}
N_{2} & =N \sum_{x=1}^{M^{\prime}} \sum_{r=0}^{M}\left(\begin{array}{c}
x+r-1 \\
r-1
\end{array}\right)[(1-\theta) p]^{r}[(1-\theta) q]^{x-1}[q(\theta)] \\
\chi & =\frac{N \sum_{x=0}^{M^{\prime}} \sum_{r=0}^{M}\left(\begin{array}{c}
x+r-1 \\
r-1
\end{array}\right)[(1-\theta) p]^{r-1}[(1-\theta) q]^{x}[p(\theta)]}{N \sum_{x=0}^{M^{\prime}} \sum_{r=0}^{M}\left(\begin{array}{c}
x+r-1 \\
r-1
\end{array}\right)[(1-\theta) p]^{r-1}[(1-\theta) q]^{x}[p(\theta)]+N \sum_{x=1}^{M^{\prime}} \sum_{r=0}^{M}\left(\begin{array}{c}
x+r-1 \\
r-1
\end{array}\right)[(1-\theta) p]^{r}[(1-\theta) q]^{x-1}[q(\theta)]}
\end{aligned}
$$

$\chi$ being the conditional probability of dying in $R_{1}$ under the dual risk of both $R_{1}$ and $R_{2}$. Hence the probability of unclassified case of death being classified and put in the category of $R_{1}$ (the exact cause of death) is given by

$$
z_{1}=\left[\chi * \frac{Q_{t-1}}{Q_{t-1}+Q_{t-2}}\right]
$$

and that of being classified in $R_{2}$ is given by

$$
z_{2}=\left[\chi * \frac{Q_{t-2}}{Q_{t-1}+Q_{t-2}}\right]
$$

where $Q_{t-1}$ is the crude probability of dying in risk $R_{1}$ in presence of risk $R_{2}$ in a duration $(0, t)$. It is given by

$$
Q_{t-1}=\int_{0}^{t} e^{-\int_{0}^{t} \lambda d \lambda} \lambda_{1} d \lambda
$$

where

$$
\begin{aligned}
\lambda & =\lambda_{1}+\lambda_{2} \\
\lambda & =\frac{\lambda_{1}}{\lambda}\left[1-e^{-\lambda t}\right] ; \\
& \cong \frac{\lambda_{1}}{\lambda}[1-(1-\lambda t)] ; \\
& \cong \frac{\lambda_{1}}{\lambda} * \lambda t .
\end{aligned}
$$

Similarly,

$$
\begin{aligned}
Q_{t-2} & =\int_{0}^{t} e^{-\int_{0}^{t} \lambda d \lambda} \lambda_{2} d \lambda, \\
\lambda & =\frac{\lambda_{2}}{\lambda}\left[1-e^{-\lambda t}\right] ; \\
& \cong \frac{\lambda_{2}}{\lambda}[1-(1-\lambda t)] ; \\
& \cong \frac{\lambda_{2}}{\lambda} * \lambda t .
\end{aligned}
$$

Following Freund [7], we make further generalization of the above parameters given that while the risk $R_{1}$ occurred with intensity $\lambda_{1}$, then the risk $R_{2}$ following $R_{1}$ occurred with greater intensity $\lambda_{2}^{\prime}$ (instead of $\left.\lambda_{2}\right)\left(\lambda_{2}^{\prime}>\lambda_{2}\right)$ : because of greater probability of the risk $R_{2}$. Similarly, with a reverse reasoning it can be shown that risk $R_{2}$ occurring earlier to $R_{1}$ will be associated with greater intensity $\lambda_{1}^{\prime}$ (instead of $\lambda_{1}$ ).
We illustrate this concept by taking Freund bivariate exponential distribution given by

$$
f(x, y)= \begin{cases}\lambda_{1} \lambda_{2}^{\prime} e^{-\lambda_{2}^{\prime} y-\left(\lambda_{1}+\lambda_{2}-\lambda_{2}^{\prime}\right) x} & \text { for } 0<x<y \\ \lambda_{2} \lambda_{1}^{\prime} e^{-\lambda_{1}^{\prime} x-\left(\lambda_{1}^{\prime}+\lambda_{2}-\lambda_{1}^{\prime}\right) y} & \text { for } 0<y<x\end{cases}
$$

We present in Table 1 the relative comparison of level of joint risks in several competing chronic conditions as follows: namely, diabetes versus heart disease, hypertension versus heart disease, chronic kidney disease versus heart disease, diabetes versus stroke, hypertension versus stroke, diabetes versus chronic kidney disease, hypertension versus chronic kidney disease, and diabetes versus hypertension.

Diabetes, hypertension, heart disease, chronic kidney disease, and stroke are not recurrent. Survival data basically are of two types, namely, (i) parallel type and (ii) series or competing type.

Parallel type of data occurs in the analysis of multicomponent parallel systems, particularly parallel paired components like two kidneys, two eyes, two lungs, and so forth. Since survival of the system depends on the longevity of the last surviving component in this case, paired data are available irrespective of whether they are censored or not.

The series types of data generally arise in case of several competing risk, say, $R_{1}, R_{2}, \ldots, R_{k}$, which may be imagined to be competing each other for taking the life of an individual or a system. But since the death or failure may occur because of a particular risk (in presence of other risk), we do not get paired or multivariate data on the longevities due to several risk bases on the same patients. Hence there exists no sample space and as such bivariate or multivariate models to measure the longevities under competing risk are not possible. This may happen more particularly in respect of requirement of paired data under the joint competing risk like diabetes with heart disease and chronic kidney disease with diabetes. Since death may occur ultimately as one of the two risks, therefore, it is not possible to get paired data or bivariate sample space.

We have considered number of correlated shocks analogous as competing risks, so the model of Biswas and Noor [3] can approximately be taken in the present investigation.

The diabetes and hypertension are the risk factors that may cause other problems that may lead to death.

Statistically, the other diseases may occur due to interaction of hypertension and diabetes. The nature of other diseases and their magnitude are unknown; therefore such interactions have been ignored assuming they will not affect the estimation. 
TABLE 1: Relative comparison of risks in several chronic conditions of mortality.

\begin{tabular}{|c|c|c|c|}
\hline Parameters & $\begin{array}{c}\text { Diabetes, } \\
\text { heart disease }\end{array}$ & $\begin{array}{c}\text { Hypertension, } \\
\text { heart disease }\end{array}$ & $\begin{array}{c}\text { Chronic kidney disease, } \\
\text { heart disease }\end{array}$ \\
\hline$p$ & 0.04 & 0.03 & 0.09 \\
\hline$q$ & 0.96 & 0.97 & 0.91 \\
\hline$N_{1}$ & 0.0007 & 0.0008 & 0.005 \\
\hline$N_{2}$ & 0.006 & 0.006 & 0.006 \\
\hline$\chi_{1}$ & 0.104 & 0.118 & 0.45 \\
\hline$\chi_{2}$ & 0.896 & 0.882 & 0.55 \\
\hline$Q_{t-1}$ & 0.06 & 0.05 & 0.14 \\
\hline$Q_{t-2}$ & 0.14 & 0.14 & 0.14 \\
\hline$Z_{1}$ & 0.03 & 0.03 & 0.23 \\
\hline$Z_{2}$ & 0.64 & 0.65 & 0.27 \\
\hline Parameters & $\begin{array}{c}\text { Diabetes, } \\
\text { chronic kidney disease }\end{array}$ & $\begin{array}{c}\text { Hypertension, } \\
\text { chronic kidney disease }\end{array}$ & $\begin{array}{c}\text { Diabetes, } \\
\text { hypertension }\end{array}$ \\
\hline$p$ & 0.04 & 0.04 & 0.04 \\
\hline$q$ & 0.96 & 0.96 & 0.96 \\
\hline$N_{1}$ & 0.0007 & 0.0008 & 0.0007 \\
\hline$N_{2}$ & 0.005 & 0.005 & 0.0008 \\
\hline$\chi_{1}$ & 0.141 & 0.16 & 0.429 \\
\hline$\chi_{2}$ & 0.877 & 0.86 & 0.571 \\
\hline$Q_{t-1}$ & 0.06 & 0.05 & 0.06 \\
\hline$Q_{t-2}$ & 0.14 & 0.14 & 0.05 \\
\hline$Z_{1}$ & 0.04 & 0.04 & 0.23 \\
\hline$\underline{Z_{2}}$ & 0.63 & 0.64 & 0.27 \\
\hline Parameters & Diabetes, stroke & $\begin{array}{c}\text { Hypertension, } \\
\text { stroke }\end{array}$ & \\
\hline$p$ & 0.04 & 0.03 & \\
\hline$q$ & 0.96 & 0.97 & \\
\hline$N_{1}$ & 0.0007 & 0.0008 & \\
\hline $\mathrm{N}_{2}$ & 0.0138 & 0.0138 & \\
\hline$\chi_{1}$ & 0.048 & 0.055 & \\
\hline$\chi_{2}$ & 0.952 & 0.945 & \\
\hline$Q_{t-1}$ & 0.06 & 0.05 & \\
\hline$Q_{t-2}$ & 0.15 & 0.15 & \\
\hline$Z_{1}$ & 0.01 & 0.01 & \\
\hline$Z_{2}$ & 0.7 & 0.72 & \\
\hline
\end{tabular}

We do not have enough data to calculate the additional joint distribution (such as heart disease with diabetes and kidney disease). The present study is devoted to joint distribution of two competing risks of mortality on the basis of relevant models. However, the methodology can be generalized on more than two competing risks.

There were $14.7 \%$ (CARRS data) individuals who have 2 or more competing risks. A substitutional proportion of $56.6 \%$ had no competing risk at baseline. In the analysis, we have considered the follow-up data of chronic conditions and mortality during the 3.5 years of follow-up.

Heart disease and chronic kidney disease have 60 to $65 \%$ chance of mortality with hypertension or diabetes. Stroke has $70 \%$ chance of mortality with hypertension or diabetes.

\section{Conditional Cause Specific Mortality Rate by Bayesian Approach}

We consider a Bayesian method of estimating the conditional probabilities of dying in heart disease and chronic kidney disease in presence of hypertension, diabetes, and stroke or heart disease with chronic kidney disease. Data consisted of $n$ individuals with observed set $S_{i}$ of binary indicators of symptoms, $S_{i}=\left(s_{i 1} ; s_{i 2} ; s_{i 3} ; s_{i 4}\right)$, and an indicator $y_{i}$ that denotes which one of $C$ causes was responsible for persons $i$ 's death.

Defining $p\left(y_{i}=c_{i} \mid S_{i}\right)$ is the conditional probability of dying in $c_{i}$ given $s_{i}$ binary system including $c_{i}$. The empirical Bayes estimator has been obtained from the prior data which 
was available from CARRS. Usually simple Bayesian estimate without prior distribution can be calculated assuming all the risks have been same probability distribution of death. Using Bayes' rule [4],

$$
\begin{aligned}
& p\left(y_{i}=c_{i} \mid S_{i}\right)=\frac{p\left(S_{i} \mid y_{i}=c\right) p\left(y_{i}=c\right)}{p\left(S_{i} \mid y_{i}=c\right) p\left(y_{i}=c\right)+p\left(S_{i} \mid y_{i} \neq c\right) p\left(y_{i} \neq c\right)} \\
& p(\text { dying in CKD | the symptoms of hypertension, diabletes or stroke }) \\
& =\frac{p(\mathrm{CKD} \mid \mathrm{CKD}, \mathrm{HTN}, \mathrm{DM}, \text { Stroke }) * p(\text { dying in CKD })}{p(\mathrm{CKD} \mid \mathrm{CKD}, \mathrm{HTN}, \mathrm{DM}, \text { Stroke }) * p(\text { dying in CKD })+p(\text { non CKD } \mid \mathrm{CKD}, \mathrm{HTN}, \mathrm{DM}, \text { Stroke }) * p(\text { not dying in CKD })} \\
& =\mathbf{0 . 3 1}
\end{aligned}
$$

$p$ (dying in Heart disease | the symptoms of hypertension, diabletes, chronic kidney disease or stroke)

$$
\begin{aligned}
& =\frac{p(\mathrm{HD} \mid \mathrm{HD}, \mathrm{CKD}, \mathrm{HTN}, \mathrm{DM}, \text { Stroke }) * p(\text { dying in HD })}{p(\mathrm{HD} \mid \mathrm{HD}, \mathrm{CKD}, \mathrm{HTN}, \mathrm{DM}, \text { Stroke }) * p(\text { dying in } \mathrm{HD})+p(\text { non HD } \mid \mathrm{HD}, \mathrm{CKD}, \mathrm{HTN}, \mathrm{DM}, \text { Stroke }) * p(\text { not dying in HD })} \\
& =\mathbf{0 . 2 5}
\end{aligned}
$$

although the Bayes method provides full information of the data and is based on sound logic of inverse probability.

\section{Conclusion}

Heart diseases (60\%), chronic kidney disease (65\%), and stroke $(70 \%)$ have higher chances of dying in the presence of hypertension and diabetes. Therefore, a future research question would be whether the individual disease is alone or it is enhanced by the competing risk.

It would be worthwhile to carry out similar studies for replications as well as enhancing an understanding of epidemiology.

\section{Disclosure}

Suddhendu Biswas was formerly the dean of Faculty of Mathematical Sciences, University of Delhi.

\section{Conflicts of Interest}

The authors have no conflicts of interest to disclose.

\section{Acknowledgments}

The CARRS Study was funded in part by the National Heart, Lung, and Blood Institute of the National Institutes of Health, Department of Health and Human Services (Contract no. HHSN268200900026C), and the United Health Group (Minneapolis, MN, USA). The CARRS Study Group includes the following: steering committee: Dorairaj Prabhakaran, KM Venkat Narayan, K Srinath Reddy, Nikhil Tandon, V Mohan, Muhammed M Kadir, and Mohammed K Ali; conduct and operations: Dorairaj Prabhakaran, Nikhil Tandon, KM Venkat Narayan, Mohammed K Ali, Roopa Shivashankar, Imran Naeem, R Pradeepa, and M Deepa; coordinating centre (Delhi): Dorairaj Prabhakaran, Nikhil Tandon, Roopa Shivashankar, Vamadevan S Ajay, and Deksha Kapoor; data management and statistical team: Dimple Kondal, Naveen, Garima, and Vandana; laboratory: Lakshmy Ramakrishnan,
Ruby Gupta, and Savita. Regarding reporting, Kalpana Singh and Professor Suddhendu Biswas had done the analysis and written the manuscript. The authors are very thankful to Professor Dorairaj Prabhakaran for editing and revising the manuscript.

\section{References}

[1] F. Downton, "Bivariate exponential distributions in reliability theory," Journal of the Royal Statistical Society. Series B (Methodological), vol. 32, no. 3, pp. 408-417, 1970.

[2] A. W. Marshall and I. Olkin, "A multivariate exponential distribution," Journal of the American Statistical Association, vol. 62, no. 317, pp. 30-44, 1967.

[3] S. Biswas and H. S. Noor, "On a probability model for classifying the unknown causes of death due to two competing risks into assignable causes," Biometrical Journal, vol. 30 , no. 7, pp. 827833,1988

[4] H. T. McCormick, Z. Richard Li, C. Calvert, C. A. Crampin, K. Kahn, and J. S. Clark, "Probabilistic cause-of-death assignment using verbal autopsies: Center for Statistics and the Social Sciences: Working Paper Series," Working Paper no. 147, 2014.

[5] World Health Organization, "STEPwise approach to surveillance (STEPS)," 2014, http://www.who.int/chp/steps/en/.

[6] M. Nair, M. K. Ali, V. S. Ajay et al., "CARRS Surveillance study: design and methods to assess burdens from multiple perspectives," BMC Public Health, vol. 12, no. 1, article 701, 2012.

[7] J. E. Freund, "A bivariate extension of the exponential distribution," Journal of the American Statistical Association, vol. 56, no. 296, pp. 971-977, 1961. 


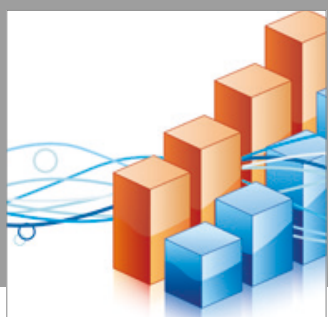

Advances in

Operations Research

vatersals

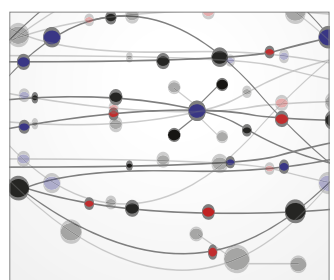

\section{The Scientific} World Journal
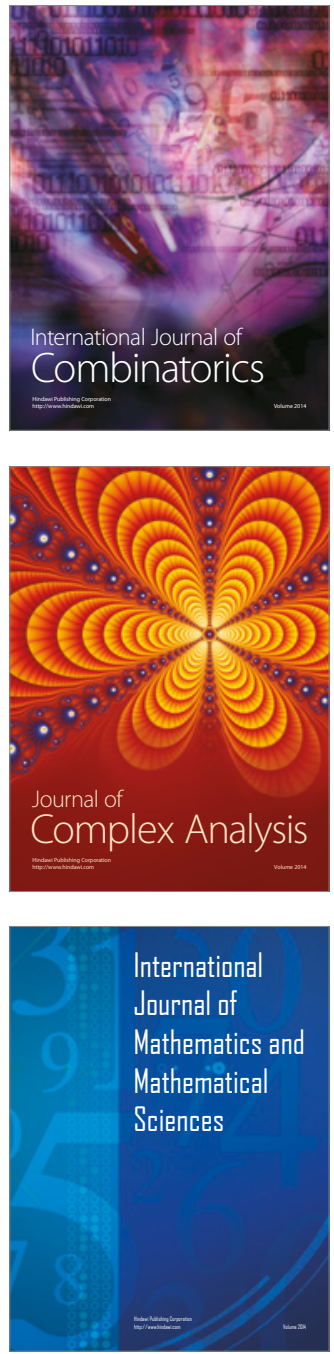
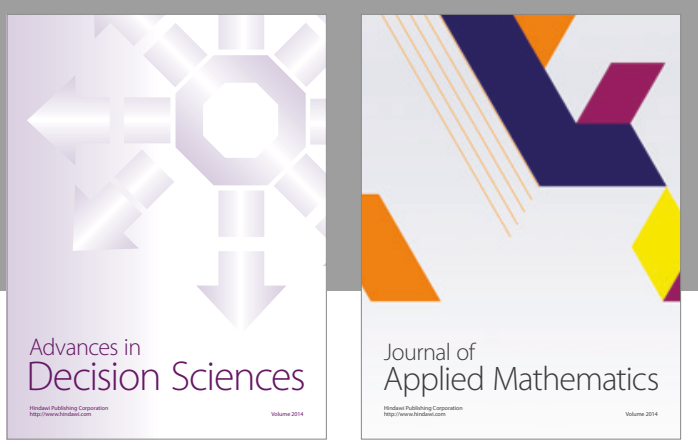

Algebra

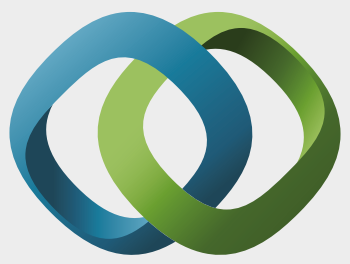

\section{Hindawi}

Submit your manuscripts at

https://www.hindawi.com
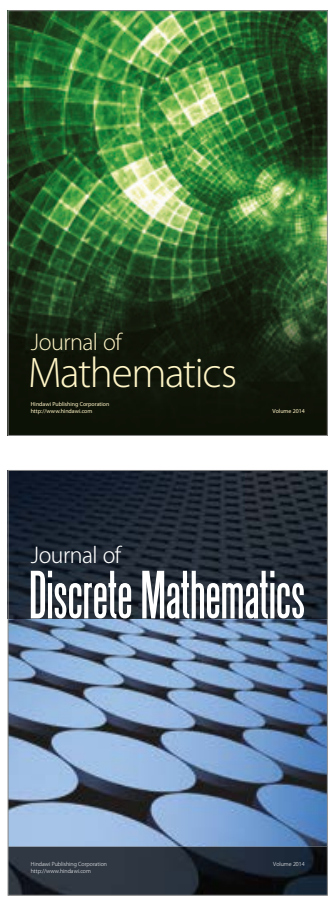

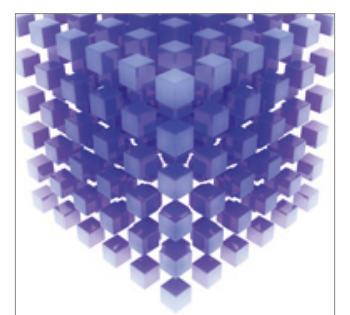

Mathematical Problems in Engineering
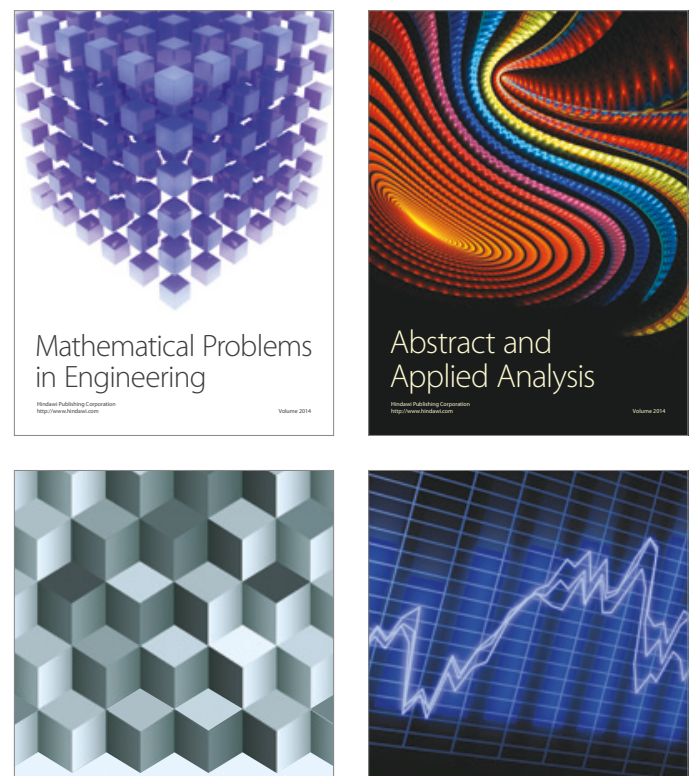

Journal of

Function Spaces

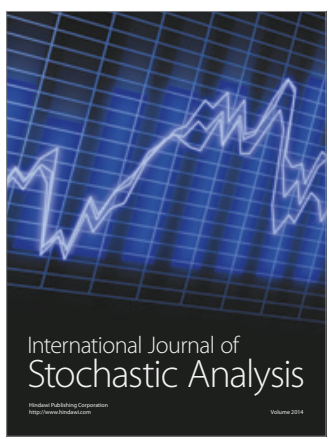

Probability and Statistics
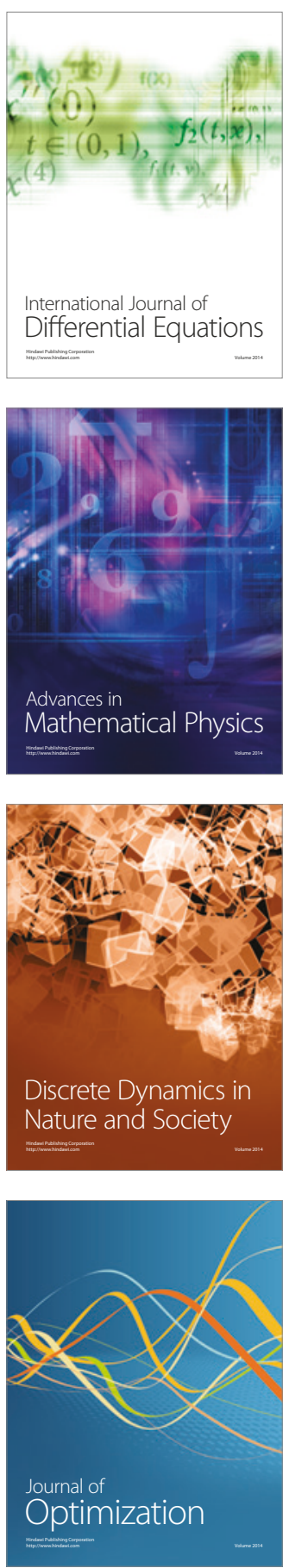